(CASE REPORT)

\title{
Acupuncture and herbal treatment for human papillomavirus (HPV) and uterine myoma
}

\author{
Zhu Jihe ${ }^{1}$, Arsovska Blagica ${ }^{1,2}$, Kozovska Kristina $1,3,{ }^{*}$ and Jovevska Svetlana 1 \\ ${ }^{1}$ Faculty of Medical Sciences, University Goce Delchev, Shtip, Republic of Macedonia. \\ ${ }^{2}$ Institute of Biology, Faculty of Natural Sciences and Mathematics, Skopje, Republic of Macedonia. \\ ${ }^{3}$ Medicine Faculty, St. Cyril and Methodius University of Skopje, Republic of Macedonia.
}

Publication history: Received on 14 May 2020; revised on 21 May 2020; accepted on 24 May 2020

Article DOI: https://doi.org/10.30574/wjarr.2020.6.2.0155

\begin{abstract}
Human papillomavirus (HPV) is the most common sexually transmitted infection globally. Almost all cervical cancers are due to HPV with two types, HPV16 and HPV18, which account for 70\% of cases. Myomas are benign growths in different parts and places of the uterus. In this research is presented a case of 32 year old woman diagnosed with uterine myoma and HPV type 16. The myoma was found in September, 2018 and the HPV infection in January, 2020, two years later after the myoma was treated. The myoma was treated with 10 acupuncture treatments, Cordiceps synensis tea capsules 1.5 grams per day and Yunnan Baiyao tea capsules 1 gram per day in a period of 4 months. The HPV treatment included 10 acupuncture treatments and 1.5 gram Cordiceps synensis tea capsules per day in a period of 3 months. The findings after the treatments for the myoma were normal, with no presence of any abnormalities. The two tests for HPV after the treatment were negative too. Acupuncture and herbal treatment as part of the TCM give very good and satisfying results in gynecological disorders and can help in resolving of conditions like HPV and myoma.
\end{abstract}

Keywords: Traditional Chinese Medicine; Acupuncture; HPV; Herbs

\section{Introduction}

Human papillomavirus (HPV) is the most common sexually transmitted infection globally. Most HPV infections do not cause any symptoms and resolve spontaneously. In some people, HPV infection persists and results in warts or precancerous lesions. Precancerous lesions increase the risk of cancer of the cervix, vagina, vulva, anus, penis, mouth or throat. Most common HPV types which cause genital infections are: type 6,12, 16, 18, 31, 33 and 35. Almost all cervical cancers are due to HPV with two types, HPV16 and HPV18, which account for 70\% of cases. HPV 6 and HPV 11 are common causes of genital warts and laryngeal papillomatosis. [1] [2]

Although most of the HPV infections and pre-cancerous lesions resolve spontaneously and clear up on their own, all women are at risk the HPV infection to become chronic and to progress into pre-cancerous lesions and invasive cervical cancer. [3] The genital types of HPV spread through sexual contact and direct contact with infected genital skin, bodily fluids and mucous membranes. [2]

Myomas are benign growths in different parts and places of the uterus. [4] Myomas or fibroids are hormonally responsive tumors, which are usually asymptomatic, but also may cause reproductive problems, pelvic pain and severe bleeding. HPV infections are known to be one of the most common causes of abnormal pap smears and cervical dysplasia. [5]

\footnotetext{
* Corresponding author: Kozovska Kristina
} 
In terms of the Traditional Chinese Medicine (TCM), fibroids are an abdominal masses produced by accumulation of Qi, blood and phlegm. [4] The HPV infection is assumed to be caused by invasion of toxins. Traditional Chinese Medicine (TCM) as a treatment options for fibroids and HPV infection uses herbal treatment and acupuncture. [6]

In TCM for the treatment of HPV and fibroids is used detoxification therapy with aim to dispel the dampness and replenish the Qi. The treatment has positive effects on the increasing of the regression rate of CIN, improving the HPV clearance and impacting the proportion of the cytokine levels and some immune cells in the genital tract. [6].

\section{Case report}

In this research is presented a case of 32 year old woman diagnosed with uterine myoma and HPV type 16 . The myoma was found in September, 2018 and the HPV infection in January, 2020, two years later after the myoma was treated.

For the myoma the patient was treated with acupuncture and was prescribed Cordiceps synensis tea capsules 1.5 grams per day and Yunnan Baiyao tea capsules 1 gram per day. The patient has made 10 treatments in a period of 4 months. The tea was taken in parallel with the treatments.

The patient had no symptoms of the HPV infection, but the finding was detected by a Pap test. Before the acupuncture treatment the patients was taking: Germanium, Imunobalance, Zinc betadine and Imuno complex plus. The patient has made 10 acupuncture treatments, once weekly in a period of three months and the Cordiceps synensis tea was taken parallel with the treatments. The patient was taking 1.5 gram Cordiceps synensis tea capsules per day.

Treatments were made in a clinic for acupuncture and Traditional Chinese Medicine by a doctor specialist in acupuncture. Treatments were made indoor, on a room temperature, with duration of 30-45 minutes each side of the body.

Acupuncture points used for the both conditions are: LR1 (Dadun), HN1 (SiShen Cong), LI4 (HeGu), ST36 (Zu San Li), GB34 (Yang Ling Quan), SP9 (Yin Ling Quan), SP6 (San Yin Jiao), LR3 (Tai Chong), RN4 (Guan Yuan), RN13 (Zhong Wan), RN2 (QuGu), RN7 (Qi Hai), ST25 (TianShu), DU14 (Da Zhui), ST29 (Gui Lai),), GB20 (Feng Chi), BL18 (Gan Shu), BL19 (DanShu), BL25 Da Chang Shu), BL21 (Wei Shu), BL32 (CiLiao).and BL20 (Pi Shu).

The findings after the treatments for the myoma were normal, with no presence of any abnormalities. On photo 1 is shown the ultrasound image done before the acupuncture and herbal treatment with clear finding of uterine myoma. On photo 2 is shown the ultrasound image done after the treatments without presence of any abnormalities.

The two tests for HPV infection after the treatment were negative too.

According to TCM, the uterine fibroids occur due to deficient spleen energy which allows dampness to accumulate, which then stagnates and becomes phlegm, congeals into a tumor in the uterus and is known as a uterine fibroid. [7]

TCM improves the clinical index in the treatment of gynecological disorders as HPV infection, fibroids, cervical cancer and genital warts; has a big impact in the regulation of cell apoptosis, the cell signal transduction pathways, the viral gene transcription and translation and the immune function. [8]

Chinese medicine regulates and treats gynecological disorders for more than 3000 years. The aim of the TCM treatment in gynecological disorders is to regulate and tonify Qi, blood, yin and yang and clear stagnation. Chinese herbal treatment is used to restore the normal organ functions and correct underlying imbalances. [9] 

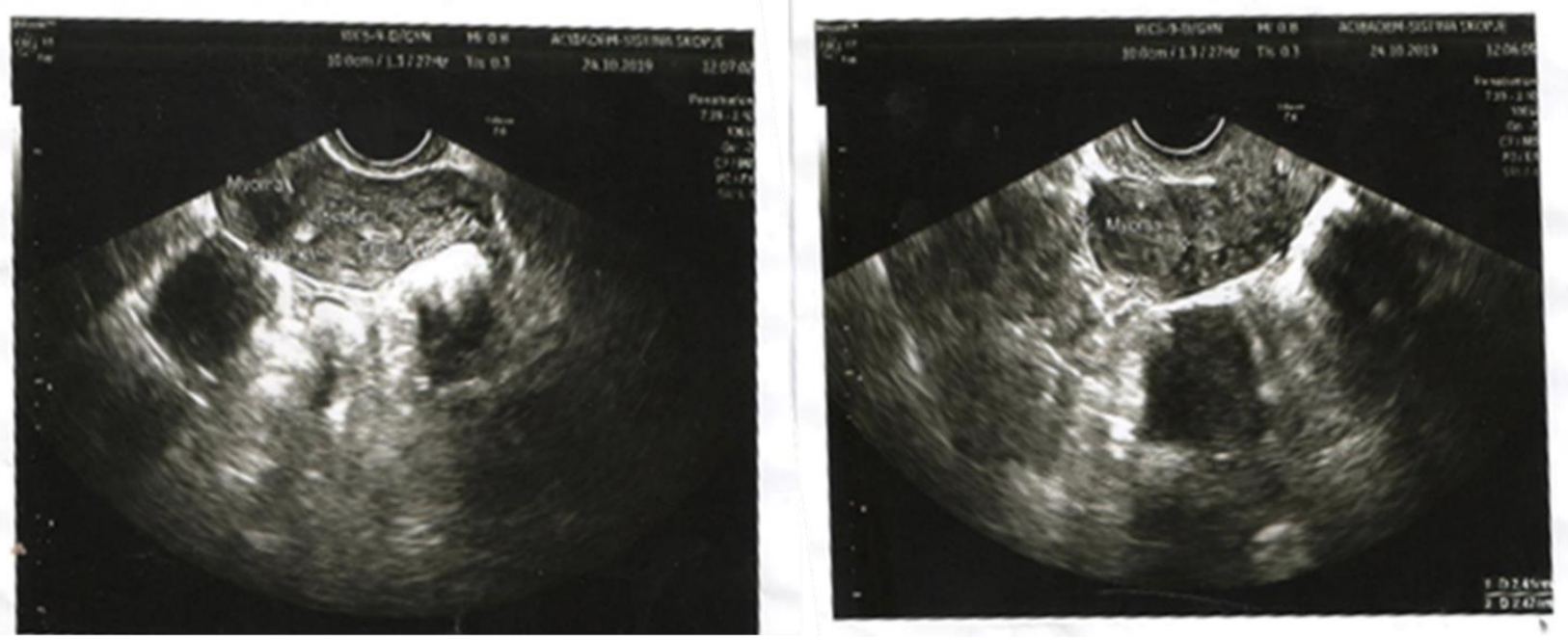

Figure 1 Ultrasound image of uterus done before the treatment
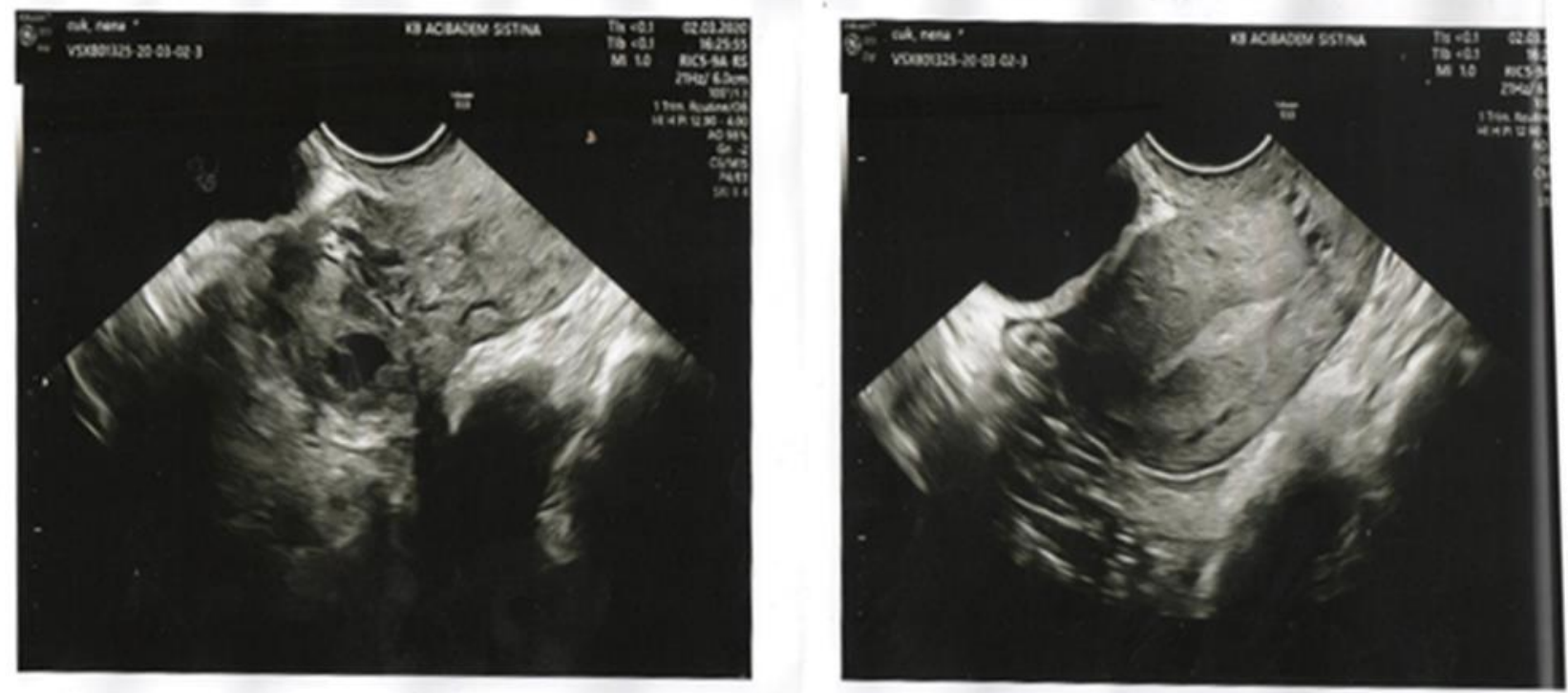

Figure 2 Ultrasound image of uterus done after the treatment

\section{Conclusion}

Acupuncture and herbal treatment as part of the TCM give very good and satisfying results in gynecological disorders and can help in resolving of conditions like HPV and myoma.

\section{Compliance with ethical standards}

\section{Disclosure of conflict of interest}

The authors declare that there is no conflict of interest.

\section{Statement of ethical approval}

In the present research work it is ensured that the dignity, rights, safety and well-being of the participant is the primary consideration of the research project. 


\section{Statement of informed consent}

Informed consent was obtained from all individual participants included in the study.

\section{References}

[1] Ninova SK. (2018). Sto pretstavuva HPV virusot.

[2] Chinese Acupuncture. (2018). TCM; HPV - What You Need to Know.

[3] Ferlay J, Ervik M, Lam F, Colombet M, Mery L, Piñeros M, Znaor A, Soerjomataram I and Bray F. (2018). Global Cancer Observatory: Cancer Today. Lyon, France: International Agency for Research on Cancer.

[4] Zhu J, Arsovska B and Kozovska K. (2010). Acupuncture and Yunnan Baiyao Treatment for Uterine Myoma; International Journal of Medical Science and Health Research; Vol. 3, No. 01; 2019 [5] Laughlin KS, Schroeder CJ, Dya Baird D; New Directions in the Epidemiology of Uterine Fibroids; Semin Reprod Med, 28(3), 204-217.

[5] Luo $M$ et al. (2019). Detoxification therapy of traditional Chinese medicine for genital tract high-risk human papillomavirus infection: A systematic review and meta-analysis; PLoS One, 14(3), e0213062.

[6] Balk LJ. (2001). Traditional Chinese Medicine and Uterine Fibroids; Integrative Medicine, 4, 43-45.

[7] Lin J et al. (2017). Traditional Chinese medicine for human papillomavirus (HPV) infections: A systematic review; Biosci Trends, 11(3), 267-273.

[8] Acupuncture \& Massage College. (2009). Traditional Chinese Medicine Gynecology.

\section{How to cite this article}

Zhu J, Arsovska B, Kozovska K and Jovevska S. (2020). Acupuncture and herbal treatment for human papillomavirus (HPV) and uterine myoma. World Journal of Advanced Research and Reviews, 6(2), 166-169. 